\title{
The superconducting phase transition and gauge dependence
}

\author{
Claude de Calan and Flavio S. Nogueira \\ Centre de Physique Théorique, Ecole Polytechnique, F-91128 Palaiseau Cedex, FRANCE
}

(Received February 10, 2017)

\begin{abstract}
The gauge dependence of the renormalization group functions of the Ginzburg-Landau model is investigated. The analysis is done by means of the Ward-Takahashi identities. After defining the local superconducting order parameter, it is shown that its exponent $\beta$ is in fact gauge independent. This happens because in $d=3$ the Landau gauge is the only gauge having a physical meaning, a property not shared by the four-dimensional model where any gauge choice is possible. The analysis is done in both the context of the $\epsilon$-expansion and in the fixed dimension approach. It is pointed out the differences that arise in both of these approaches concerning the gauge dependence.
\end{abstract}

Pacs: 74.20.-z, 05.10Cc, 11.10.-z

Eltitzur's theorem [1] states that a local gauge symmetry cannot be sponteneously broken. As a consequence, no local order parameter can be defined in a model described by a locally gauge invariant action. However, the gauge symmetry can be explicitly broken, for instance, by adding a gauge fixing term to the action. In the context of the usual lattice Ginzburg-Landau (GL) model [3] but with a gauge fixing term, Kennedy and King [2] have proposed the following non-local order parameter (OP):

$$
G_{\infty}=\lim _{|\mathbf{x}-\mathbf{y}| \rightarrow \infty}<G(\mathbf{x}, \mathbf{y})>
$$

where the operator $G(\mathbf{x}, \mathbf{y})$ is given by a smeared string:

$$
G(\mathbf{x}, \mathbf{y})=\phi_{\mathbf{x}} \exp \left[-i e \sum_{\mu, \mathbf{z}} A_{\mu \mathbf{z}} h_{\mu \mathbf{z}}\right] \phi_{\mathbf{y}}^{*},
$$

where $\phi_{\mathbf{x}}$ and $A_{\mu \mathbf{x}}$ are the scalar and gauge fields, respectively, defined in a lattice. The gauge group is noncompact. The field $h_{\mu \mathbf{z}}=\Delta_{\mu} V_{\mathbf{z}-\mathbf{x}}-\Delta_{\mu} V_{\mathbf{z}-\mathbf{y}}$, with $\Delta_{\mu}$ being a lattice derivative and $V$ is the kernel of $(-\bar{\Delta})^{-1}$, where $\bar{\Delta}$ is the lattice Laplacian. The operator $G$ so defined is gauge invariant and in the Landau gauge $\sum_{\mu, \mathbf{z}} A_{\mu \mathbf{z}} h_{\mu \mathbf{z}}$ vanishes [2]. By using the so defined order parameter, Kennedy and King have shown that there is true long range order for $d \leq 4\left(G_{\infty} \neq 0\right)$ only if the Landau gauge is fixed. Note that this result was proved for a lattice GL model only and is not a trivial matter to extend the analysis of Ref. [2] to the continuum. The aim of this report is to provide an analysis of the gauge dependence directly in the continuum. Also, we will define a local, gauge invariant OP. To this end, we will employ the Ward-Takahashi (WT) identities. The study that will be undertaken here was initiated recently by one of us [4].

Let us consider the following bare action for the GL model:

$$
\begin{aligned}
S & =\int d^{d} x\left[\frac{1}{4} F_{0}^{2}+\left(D_{\mu}^{0} \phi_{0}\right)^{\dagger}\left(D_{\mu}^{0} \phi_{0}\right)+\frac{M_{0}^{2}}{2} A_{\mu}^{0} A_{\mu}^{0}\right. \\
& \left.+m_{0}^{2}\left|\phi_{0}\right|^{2}+\frac{u_{0}}{2}\left|\phi_{0}\right|^{4}\right]+S_{g f},
\end{aligned}
$$

where the zeroes denote bare quantities, $F_{0}^{2}$ is a short for $F_{0}^{\mu \nu} F_{0}^{\mu \nu}$ and $D_{\mu}^{0}=\partial_{\mu}+i e_{0} A_{\mu}^{0}$. The $S_{g f}$ is the gauge fixing part and is given by

$$
S_{g f}=\int d^{d} x \frac{1}{2 a_{0}}\left(\partial_{\mu} A_{\mu}^{0}\right)^{2}
$$

The mass term for the gauge field is introduced in order to avoid infrared divergences. The renormalized counterpart of $M_{0}$ tends to zero as approaching the critical point more rapidly than $m$ [4] (quantities without zeroes are renormalized quantities. Renormalization is done in a standard way [6] and the renormalization conditions are the same as in Ref. [4). We note that only averages of gauge invariant operators have a physical meaning. Thus, although $\langle\phi\rangle$ could be different from zero due to the presence of terms in the action which break explictly gauge invariance, this average is surely gauge dependent. Only averages of gauge invariant quantities are gauge independent 6, 12]. For instance, the superfluid density $\rho_{s}=<|\phi|^{2}>$ is gauge independent. We can write

$$
\rho_{s}=<|\phi|^{2}>=Z_{\phi}^{-1}<\left|\phi_{0}\right|^{2}>.
$$

where $Z_{\phi}$ is the wave function renormalization of the scalar field. Near the critical point we have $Z_{\phi} \sim|m|^{\eta} \sim$ $|t|^{\nu \eta}$, where $t$ is the reduced temperature and $\eta$ is the fixed point value of the renormalization group (RG) function $m \partial \ln Z_{\phi} / \partial m$ (we assume the existence of an infrared stable fixed point; for a discussion on this point see Refs. [10,7,5,8,9] and references therein). Note that the critical exponents should be the same above and below $T_{c}$. This is a consequence of the fact that the same counterterms of the symmetric phase can be used when renormalizing the broken symmetry theory if a minimal subtraction is used. This fact is a consequence of the WT identities [6, 12]. $Z_{\phi}$ is a gauge dependent quantity (by gauge dependence here we mean dependence on $a$ and not on $a_{0}$ ). This means that $<\left|\phi_{0}\right|^{2}>$ should depend on $a$ in such a way as to cancel the gauge dependence of $Z_{\phi}^{-1}$. Note that $<\left|\phi_{0}\right|^{2}>$ does not depend on $a_{0}$ since it is a gauge invariant quantity. It is easy to show that $\left\langle\left|\phi_{0}\right|^{2}>\sim|t|^{\nu(d-2+\eta)}\right.$ near 
the critical point. This scaling suggests the following definition of a local OP:

$$
\Phi=\sqrt{<\left|\phi_{0}\right|^{2}>},
$$

having a critical exponent $\beta=\nu(d-2+\eta) / 2$. In order to prove that $\beta$ is gauge independent we need to prove that $\eta$ is gauge independent. Note that the other renormalized parameters of the model are trivially gauge independent. This statement implies the gauge independence of all beta functions (which does not mean necessarily that the bare parameters are gauge independent).

In order to obtain the gauge dependence of $Z_{\phi}$ we will employ the following WT identity:

$$
\begin{aligned}
\left(M^{2}-\frac{1}{a} \partial_{z}^{2}\right) \partial_{\mu}^{z} W_{\mu}^{(2)}(z ; y, x) & =i e\left[\delta(y-z) W^{(2)}(z, x)\right. \\
& \left.-\delta(z-x) W^{(2)}(y, z)\right] .
\end{aligned}
$$

By using twice Eq. (7) we obtain

$$
\begin{aligned}
W_{\left(\partial_{\mu} A_{\mu}\right)^{2}}^{(2)}(p) & =2 e^{2} \int \frac{d^{d} k}{(2 \pi)^{3}} \frac{a^{2}}{\left(k^{2}+a M^{2}\right)^{2}}\left[W^{(2)}(p+k)\right. \\
& \left.-W^{(2)}(p)\right]
\end{aligned}
$$

where $W_{\left(\partial_{\mu} A_{\mu}\right)^{2}}^{(2)}(p)$ is the Fourier transform of

$$
\begin{aligned}
W_{\left(\partial_{\mu} A_{\mu}\right)^{2}}^{(2)}(x, y) & =\int d^{d} z\left[<\left(\partial_{\mu} A_{\mu}\right)^{2}(z) \phi(x) \phi^{\dagger}(y)>\right. \\
& \left.-<\left(\partial_{\mu} A_{\mu}\right)^{2}(z)><\phi(x) \phi^{\dagger}(y)>\right] .
\end{aligned}
$$

Let us denote the bare counterpart of $W_{\left(\partial_{\mu} A_{\mu}\right)^{2}}^{(2)}$ by $W_{\left(\partial_{\mu} A_{\mu}^{0}\right)^{2}, 0}^{(2)}$. We have that

$$
2 a_{0}^{2} \frac{\partial W_{0}^{(2)}}{\partial a_{0}}(x, y)=W_{\left(\partial_{\mu} A_{\mu}^{0}\right)^{2}, 0}^{(2)}(x, y),
$$

where $W_{0}^{(2)}(x, y)=<\phi_{0}(x) \phi_{0}^{\dagger}(y)>$ is the bare 2-point connected correlation function. Eq. (8) is valid also if we replace the renormalized correlation functions by the bare ones and the renormalized couplings by their bare counterparts. Using then a bare version of (8) and Eq. (10), we obtain

$$
\frac{\partial W_{0}^{(2)}}{\partial a_{0}}(p)=e_{0}^{2} \int \frac{d^{3} k}{(2 \pi)^{3}} \frac{W_{0}^{(2)}(p+k)-W_{0}^{(2)}(p)}{\left(k^{2}+a_{0} M_{0}^{2}\right)^{2}} .
$$

Eq. (11) can be rewritten as

$$
\begin{array}{r}
\frac{\partial \ln Z_{\phi}}{\partial a_{0}} W^{(2)}(p)+\frac{\partial W^{(2)}}{\partial a_{0}}(p)= \\
e_{0}^{2} \int \frac{d^{3} k}{(2 \pi)^{3}} \frac{W^{(2)}(p+k)-W^{(2)}(p)}{\left(k^{2}+a_{0} M_{0}^{2}\right)^{2}},
\end{array}
$$

out of which we obtain

$$
\begin{aligned}
\frac{\partial \ln Z_{\phi}}{\partial a_{0}} & =-e_{0}^{2} \int \frac{d^{d} k}{(2 \pi)^{d}} \frac{1}{\left(k^{2}+a_{0} M_{0}^{2}\right)^{2}} \\
& =e_{0}^{2} a_{0}^{\frac{d-4}{2}} M_{0}^{d-4}\left(\frac{d}{2}-1\right) C_{d},
\end{aligned}
$$

where $\pi / C_{d}=(4 \pi)^{d / 2} \Gamma(d / 2) \sin (\pi d / 2)$. Note that we have a pole for $d=4$ in the second line of Eq. (13). This is a consequence of the logarithmic divergence for $d=4$. In the $\epsilon$-expansion the singular part of the different correlation functions is isolated as poles in $1 / \epsilon$ with $\epsilon=4-d$ and the renormalization constants are written as power series in $1 / \epsilon$. This way of doing the things leads to the determination of the critical exponents as power series in $\epsilon$ [6]. The physical case of interest in critical phenomena of superfluid and magnetic systems corresponds to $\epsilon=1$.

By integrating the first line of (13) we obtain

$\ln Z_{\phi}\left(a_{0}\right)=\ln Z_{\phi}\left(a_{0}=0\right)-e_{0}^{2} a_{0} \int \frac{d^{d} k}{(2 \pi)^{d}} \frac{1}{k^{2}\left(k^{2}+a_{0} M_{0}^{2}\right)^{2}}$.

Since $e_{0}^{2} a_{0}=e^{2} a$ and $a_{0} M_{0}^{2}=a M^{2}$, we can rewrite Eq. (14) as

$$
\ln Z_{\phi}(a)=\ln Z_{\phi}(a=0)-e^{2} a \int \frac{d^{d} k}{(2 \pi)^{d}} \frac{1}{k^{2}\left(k^{2}+a M^{2}\right)^{2}} .
$$

Let us assume that $Z_{\phi}(a=0)$ has been evaluated as a power series in $1 / \epsilon$. After regularizing dimensionally the integral in (15), we obtain

$$
\eta_{\phi}(a)=\eta_{\phi}(a=0)-\frac{a f}{2 \pi},
$$

which gives the gauge dependence of $\eta_{\phi}$ in the framework of the $\epsilon$-expansion $\left(f=e^{2} m^{-\epsilon}\right)$. Let us assume that an infrared stable fixed point has been obtained, for instance, by ressummation methods [5]. As $m \rightarrow 0$, $f \rightarrow f_{*} \neq 0$ (if $\epsilon=1$ ), but $a$ scales as $m^{-1}$ near the fixed point and any non-zero $a$ runs away as $m \rightarrow 0$. Thus, the only safe way towards the charged fixed point is over the line $a=0$, that is, the Landau gauge. Note that for the case of interest in particle physics, $d=4$, we obtain the same equation as (16). However, for $d=4$ any gauge choice is possible since $f_{*}=0$ in this case.

In the fixed dimension approach things work differently. For $d=3$ the integral in Eq. (15) is convergent and we can interchange the differentiation with respect to $\mathrm{m}$ with the integral sign. Since $a e^{2}$ and $a M^{2}$ are both RG invariants, we obtain

$$
\eta_{\phi}(a)=\eta_{\phi}(a=0)
$$

and we obtain again that the physical gauge corresponds to $a=0$. At this point some remarks are in order. First, from Eq. (17) we obtain $\partial \eta_{\phi} / \partial a=0$ while the same is not true for the $\eta_{\phi}(a)$ given in Eq. (16). Second, 
Eq. (17) can be easily checked at 1-loop order. Concerning the 1-loop example, it is instructive to ask ourselves what happens in other fixed dimension approaches. For instance, we could perform a critical point $(m=0)$ calculation where the renormalization conditions are defined at non-zero external momenta, taking the symmetrical point for functions which depend on more than one momentum variable [10,11]. In this case the photon mass $M$ is unecessary since the non-zero external momenta take care of infrared divergences. The 1-loop expression for $Z_{\phi}$ in an arbitrary gauge is in this case rather simple and has been calculated by Schakel [13]. It turns out in this case that $Z_{\phi}$ is independent of $a$ if $d=3$.

The gauge dependence of $Z_{m}$ can be obtained in an analogous way. From $m^{2}=Z_{\phi} Z_{m}^{-1} m_{0}^{2}$, we obtain that $W_{0}^{(2)}(0)=Z_{m} / m_{0}^{2}$. Using again the bare version of (8), we obtain exactly the same equation as Eq. (13) but with $Z_{\phi}$ replaced by $Z_{m}$. This means that the gauge dependence of $Z_{m}$ is the same as for $Z_{\phi}$. If we use the $\epsilon$-expansion we have that $\eta_{m}-\eta_{\phi}$ is gauge independent since the gauge dependence of $\eta_{m}$ will cancel exactly the gauge dependence of $\eta_{\phi}$. In fixed dimension $d=3$, on the other hand, $\eta_{m}(a)=\eta_{m}(a=0)$. It follows that the critical exponent $\nu$ is gauge independent. Since $\eta$ is gauge independent, it follows that $\beta$ is gauge independent and the order parameter $\Phi$ has a true physical meaning.

[1] S. Elitzur, Phys. Rev. D 12, 3978 (1975).

[2] T. Kennedy and C. King, Phys. Rev. Lett. 55, 776 (1985); Commun. Math. Phys. 104, 327 (1986); C. Borgs and F. Nill, ibidem, page 349.

[3] H. Kleinert, Gauge Fields in Condensed Matter, vol. 1, World Scientific, 1993.

[4] F. S. Nogueira, Europhys. Lett. 45, 612 (1999).

[5] R. Folk and Y. Holovatch, J. Phys. A 29, 3409 (1996).

[6] J. Zinn-Justin, Quantum Field Theory and Critical Phenomena, 2nd edition (Oxford, 1993).

[7] M. Kiometzis, H. Kleinert and A. M. J. Schakel, Phys. Rev. Lett. 73, 1975 (1994).

[8] Z. Tesanovic, Phys. Rev. B 59, 6449 (1999).

[9] C. de Calan and F. S. Nogueira, Phys. Rev. B 60, to appear August 1st, 1999.

[10] I. F. Herbut and Z. Tesanović, Phys. Rev. Lett. 76, 4588 (1996); I. D. Lawrie, Phys. Rev. Lett., 78, 979 (1997); I. F. Herbut and Z. Tesanović, Phys. Rev. Lett. 78, 980 (1997).

[11] C. de Calan, A. P. C. Malbouisson, F. S. Nogueira and N. F. Svaiter, Phys. Rev. B 59, 554 (1999).

[12] J. C. Collins, Renormalization (Cambridge, 1984).

[13] A. M. J. Schakel, cond-mat/9805152 (unpublished). 